\title{
The association between coffee and caffeine consumption and renal function: insight from individual-level data, Mendelian randomization, and meta-analysis
}

\author{
Mohsen Mazidi ${ }^{1}$, Dimitri P. Mikhailidis², Abbas Dehghan³ ${ }^{3}$ Jacek Jóźwiak ${ }^{4}$, Adrian Covic ${ }^{5}$, \\ Naveed Sattar ${ }^{6}$, Maciej Banach ${ }^{7,8}$ on behalf of the Lipid and Blood Pressure Meta-analysis \\ Collaboration (LBPMC) Group and the International Lipid Expert Panel (ILEP)
}

\begin{abstract}
'Department of Twin Research and Genetic Epidemiology, King's College London, St Thomas' Hospital, Strand, London, UK

2Department of Clinical Biochemistry, Royal Free Campus, University College London Medical School, University College London (UCL), London, UK

${ }^{3} \mathrm{MRC}-\mathrm{PHE}$ Centre for Environment and Health, School of Public Health, Imperial College London, London, UK

${ }^{4}$ Department of Family Medicine and Public Health, Institute of Medicine, University of Opole, Opole, Poland

${ }^{5}$ Department of Nephrology, “Dr. C. I. Parhon” Clinical Hospital Iasi, Iasi, Romania ${ }^{6}$ Institute of Cardiovascular and Medical Sciences, University of Glasgow, Glasgow, UK ${ }^{7}$ Cardiovascular Research Centre, University of Zielona Gora, Zielona Gora, Poland ${ }^{8}$ Department of Preventive Cardiology and Lipidology, Medical University of Lodz, Lodz, Poland
\end{abstract}

Submitted: 2 December 2021; Accepted: 14 December 2021

Online publication: 14 December 2021

Arch Med Sci 2022; 18 (4): 900-911

DOI: https://doi.org/10.5114/aoms/144905

Copyright (c) 2022 Termedia \& Banach

\section{Abstract}

Introduction: The reported relationship between coffee intake and renal function is poorly understood. By applying two-sample Mendelian randomization (MR) and systematic review and meta-analysis we investigated the association of caffeine and coffee intake with prevalent CKD and markers of renal function.

Material and methods: For the individual data analysis we analyzed the National Health and Nutrition Examination Surveys (NHANES) data on renal function markers and caffeine intake. MR was implemented by using summary-level data from the largest ever genome-wide association studies (GWAS) conducted on coffee intake $(N=91,462)$ and kidney function $(N=133,413)$. The inverse variance weighted method (IVW), weighted median-based method, MR-Egger, MR-RAPS, and MR-PRESSO were applied. Random effects models and generic inverse variance methods were used to synthesize quantitative and pooled data for the meta-analysis, followed by a leave-one-out method for sensitivity analysis.

Results: Finally, we included the data of 18,436 participants; $6.9 \%$ had prevalent CKD (based on eGFR). Caffeine intake for the general population was $131.1 \pm 1.1 \mathrm{mg}$. The percentage of participants with CKD, by caffeine quartile, was $16.6 \%$ in the first (lowest) quartile, $13.9 \%$ in the second, $12.2 \%$ in the third and $11.0 \%$ in the top quartile $(p<0.001)$. After adjustment, for increasing quartiles for caffeine consumption, mean urine albumin, albumin-creatinine ratio and estimated glomerular filtration rate (GFR) did not change significantly $(p>0.234)$. In fully adjusted logistic regression models, there was no significant difference in chances of CKD prevalence ( $p$-trend $=0.745$ ). In the same line, the results of MR showed no impact of coffee intake on

\author{
Corresponding authors: \\ Dr. Mohsen Mazidi \\ Department of Twin \\ Research and \\ Genetic Epidemiology \\ King's College London \\ St Thomas' Hospital \\ Strand, London \\ SE1 7EH, UK \\ Phone: +8613167518660 \\ E-mail: mohsen.mazidi@kcl. \\ ac.uk
}

Prof. Maciej Banach

MD, PhD, FNLA, FAHA,

FESC, FASA

Department of

Preventive Cardiology

and Lipidology

Medical University

of Lodz

281/289 Rzgowska St

93-338 Lodz, Poland

E-mail: maciej.banach@

umed.lodz.pl 
CKD (IVW: $\beta=-0.0191, \mathrm{SE}=0.069, p=0.781$ ) or on eGFR (overall $=\mathrm{IVW}: \beta=-0.0005$, $\mathrm{SE}=0.005, p=0.926$ ) either in diabetic (IVW: $\beta=-0.006, \mathrm{SE}=0.009, p=0.478$ ) or non-diabetic patients (IVW: $\beta=-6.772$, $\mathrm{SE}=0.006, p=0.991)$. Results from the meta-analysis indicated that coffee consumption was not significantly associated with CKD (OR $=0.85,95 \% \mathrm{Cl}: 0.71-1.02, p=0.090, n=6$ studies, $\left.I^{2}=0.32\right)$. These findings were robust in sensitivity analyses.

Conclusions: Implementing different strategies, we detected no significant association between coffee consumption and renal function or risk of CKD.

Key words: coffee, chronic kidney disease, Mendelian randomization, NHANES, systematic review.

\section{Introduction}

Chronic kidney disease (CKD) is a progressive loss of renal function over time, ultimately leading to end-stage renal disease, which means irreversible renal failure [1]. The pathophysiological processes involved in CKD development are characterized by a background of low-grade chronic inflammation and a high level of oxidative stress [2]. Together with coagulation disorders and neutrophil-endothelium interaction, inflammation is believed to play a role in the genesis of kidney injury, potentially leading to chronically impaired kidney function [3]. According to the available data, it has been confirmed that diet might play a central role in the regulation of chronic inflammation and anti-oxidant balance, and might essentially improve kidney functions [4-6].

Caffeinated drinks are very commonly consumed mainly in Western countries [7]. It is estimated that more than $50 \%$ of US adults drink coffee on a daily basis [7]. Epidemiological studies and experimental research suggests that coffee consumption may help to prevent several chronic conditions, including type 2 diabetes (DMT2), liver disease, and cardiovascular disease (CVD) [7, 8]. With regard to the impact of coffee drinking on CKD, available results have been inconsistent [813]. Several studies have reported no significant relationship between coffee consumption and likelihood of CKD [8, 11, 12], while others have reported an inverse association [9, 10, 13]. The interpretation of single studies to date has been limited by sample size, research design and subject traits (gender, ethnicity, age, etc.), and they have been underpowered to be able to provide a comprehensive and reliable conclusion. Nakajima et al. in their study with 4722 participants aged 26-65 years and followed up for 15 years found that coffee consumption was associated with a modest increase in estimated glomerular filtration rate (eGFR) [11]. It has been hypothesized that various components of coffee may preserve the glomerular endothelial cells from oxidative stress and inflammation [8].

When considering the available data we are not able to rule out the chance of residual bias, confounding factors, and reverse causation. Mende- lian randomization (MR) analysis is able to circumvent the limitations of observational studies, and circumvent the residual bias, confounding factors and reverse causation by using genetic variants that are associated with an exposure as instruments (= coffee) to test for associations with an outcome (= renal function) [14].

Taking all the above into account, we aimed to resolve this uncertainty by investigating the association between caffeine intake, renal function, assessed by biochemical indices, and prevalent CKD in adult Americans, by conducting MR analysis on the recognized single nucleotide polymorphisms (SNPs) of coffee consumption and their causal impact on renal function, and systematically reviewing the literature and performing a meta-analysis.

\section{Material and methods}

\section{NHANES population data}

We used data from the Nutrition and Health Examination Surveys (NHANES), which is described in detail elsewhere [15]. In brief, these are periodic cross-sectional surveys conducted by the US National Center for Health Statistics (NCHS), and during which home visits are conducted to administer questionnaires to collect data on demographics, diet, and other health behaviors. NHANES applies complex multistage probability sampling procedure to ensure selection of participants from various geographical locations and adequate racial/ethnic representation [15]. Trained interviewers collected participants' demographic, socioeconomic, dietary, and health-related information using questionnaires administered during home visits. Clinical examination and dietary assessment are conducted by skilled personnel using a mobile examination center (MEC) [15]. All procedures were carried out in accordance with relevant approved guidelines and regulations [16-19]. Informed consent was obtained from all participants, and the NCHS Research Ethics Review Board approved the protocol.

For the present analysis, four survey cycles (between 2005 and 2010) were combined to produce estimates with greater precision and a smaller sampling error. The analytical sample was limited to adults aged $\geq 18$ years. After excluding 
Mohsen Mazidi, Dimitri P. Mikhailidis, Abbas Dehghan, Jacek Jóźwiak, Adrian Covic, Naveed Sattar, Maciej Banach

on behalf of the Lipid and Blood Pressure Meta-analysis Collaboration (LBPMC) Group and the International Lipid Expert Panel (ILEP)

pregnant and lactating women $(n=865)$, as well as participants with missing information on the variables of interest $(n=2935)$, the final analytical sample included 18,436 respondents from NHANES 2005-2010.

Smoking was based on self-report. The poverty to income variable is an index for the ratio of family income to poverty. The Department of Health and Human Services' (HHS) poverty guidelines were used as the poverty measure to calculate this index [19]. For the assessment of height and weight during the physical examination, participants were dressed in underwear, disposable paper gowns and foam slippers. Body mass index (BMI) was calculated as weight, in $\mathrm{kg}$, divided by the square of height, in meters [19]. Three and sometimes four BP (including systolic (SBP) and diastolic blood pressure (DBP)) measurements were taken using a mercury sphygmomanometer by a physician.

\section{Biochemical analysis}

Methods for biochemical analyses are described in detail in the NHANES Laboratory/Medical Technologists Procedures Manual [16-19]. A blood specimen was drawn from the participant's antecubital vein by a trained phlebotomist according to a standardized protocol. Fasting glucose was measured in plasma by a hexokinase method using a Roche/Hitachi 911 Analyzer and Roche Modular P Chemistry Analyzer. The DxC800 modular chemistry side uses the Jaffe reaction method (kinetic alkaline picrate) to determine the concentration of creatinine in serum. The creatinine calibration is traceable to an isotope dilution mass spectrometry reference method [20]. Urinary creatinine by the Jaffe rate reaction, and urinary albumin by solid-phase fluorescent immunoassay, from a random urine sample [21], were used to calculate the urinary albumin-creatinine ratio (ACR). The CKD Epidemiology Collaboration (CKD-EPI) equation [22] was used to estimate glomerular filtration rate (eGFR, in $\left.\mathrm{ml} / \mathrm{min} / 1.73 \mathrm{~m}^{2}\right)$, and an eGFR of $<60 \mathrm{ml} / \mathrm{min} / 1.73 \mathrm{~m}^{2}$ was used to define low eGFR. ACR $>30 \mathrm{mg} / \mathrm{g}$ was used to define albuminuria, and the presence of either low eGFR or albuminuria was used to define CKD in line with the Kidney Disease: Improving Global Outcomes (KDIGO) 2012 recommendations [23]. Levels of triglyceride (TG) were measured enzymatically. DMT2 was diagnosed as a self-reported history of diabetes or fasting plasma glucose $\geq 126 \mathrm{mg} / \mathrm{dl}$. Hypertension (HTN) was diagnosed in individuals with SBP at or above $140 \mathrm{~mm} \mathrm{Hg}$ and/or DBP at or above $90 \mathrm{~mm} \mathrm{Hg}$, and in persons who were on anti-hypertensive therapy [24].

Dietary data in NHANES were collected using a single 24-hour dietary recall interview at the MEC
[15]. Dietary intake was assessed via $24 \mathrm{~h}$ recall obtained by a trained interviewer during the mobile examination center visit, using a computer-assisted dietary interview system with standardized probes, i.e. the US Department of Agriculture Automated Multiple-Pass Method (AMPM) [25, 26]. Briefly, the type and quantity of all foods and beverages consumed in a single $24 \mathrm{~h}$ period before the dietary interview (from midnight to midnight) were collected using the AMPM. The AMPM is designed to enhance complete and accurate data collection while reducing respondent burden $[26,27]$. Caffeine concentration data reported in the survey-specific Food and Nutrient Database for Dietary Studies (FNDDS) were relied upon in the analyses. FNDDS is the database that provides the nutrient values for foods and beverages reported in What We Eat in America (WWEIA), the dietary intake component of NHANES. Sources of caffeine included coffee, tea, soda, energy drinks, and chocolate and cocoa containing products, in consideration of caffeinated and decaffeinated versions [28].

\section{Statistical analysis}

Data analyses followed the CDC guidelines for complex NHANES data analysis, accounting for the masked variance and using the recommended weighting methodology [29], implemented using SPSS complex sample module version 22.0 (IBM Corp, Armonk, NY). We used means and standard error of the mean (SEM) for continuous variables (with groups compared via analysis of variance (ANOVA)) and percentages for categorical variables (with groups compared using the $\chi^{2}$ test). The natural logarithm of ACR and urinary albumin were taken to approximate a normal distribution. Adjusted mean of kidney function markers (urine albumin, serum creatinine, ACR, eGFR) across caffeine quartiles were conducted using analysis of covariance (ANCOVA). These models were adjusted for age, sex, race, income to poverty, alcohol intake, energy intake, smoking, BMI, HTN, TG and T2DM. Logistic regressions models, employing a similar adjustment strategy (age, sex, race, income to poverty, alcohol intake, energy intake, smoking, BMI, HTN, TG and T2DM), were then used to derive the odds ratio (OR) and 95\% confidence interval $(\mathrm{Cl})$ for the association of caffeine (by quartile) with prevalent CKD, always using the lowest quartile as a reference. A two-sided $p<$ 0.05 was used as the nominal cut-point to indicate statistically significant results.

\section{Mendelian randomization}

\section{Study design}

A two-sample MR study design was used, in which summary statistics from different ge- 
nome-wide association studies (GWAS) were analyzed, for the exposures and outcomes, to estimate the effects of exposure on outcome. Essentially, we applied genetic predictors of coffee consumption to extensively genotyped case-control studies of kidney function (CKD, eGFR (separately in patients with and without DM)) to obtain estimates of the association of exposure with our clinical outcomes.

\section{Genetic predictors of exposures}

We retrieved summary data for the association between SNPs and coffee consumption from the biggest meta-GWAS $(91,462$, European descent, Coffee and Caffeine Genetics Consortium) [28] (Supplementary Table SI). 10 single nucleotide polymorphisms (SNPs), at eight loci, were identified as associated with coffee consumption [30]; we excluded three SNPs that did not exceed the genome-wide significance threshold $\left(p<5 \times 10^{-8}\right)$ and two SNPs in linkage disequilibrium $\left(r^{2}>0.6\right)$ leaving five independent SNPs for the main analyses. The MR studies assume that the SNPs (instrumental variables) are associated with the outcome only via the exposure [31], so we performed sensitivity analysis excluding SNPs with potentially pleiotropic effects. To assess the instrumental variable analysis "exclusion-restriction" assumption we used Ensembl release (http://useast.ensembl. org/index.html). Ensembl gives SNP phenotypes.

\section{Genetic predictors of outcomes}

Genetic associations with renal function were obtained from the largest available extensively genotyped study based on a meta-analysis $(133,413$ individuals with replication in up to 42,166 individuals) [32]. eGFR was estimated using the four-variable Modification of Diet in Renal Disease Study Equation. They defined CKD as eGFR $<60 \mathrm{ml} / \mathrm{min}$ per $1.73 \mathrm{~m}^{2}$. Diabetes was defined as fasting glucose $\geq 126 \mathrm{mg} / \mathrm{dl}$, pharmacologic treatment for diabetes or by self-reports. In all studies, diabetes and kidney function were assessed at the same point in time. For genome-wide association analysis they followed a centralized analysis plan. Each study regressed sex and age adjusted residuals of the logarithm of eGFR on SNP dosage levels. Logistic regression of CKD status was performed on SNP dosage levels adjusting for sex and age. For all traits, adjustment for appropriate study-specific features, including study site and genetic principal components, was included in the regression and family-based studies appropriately accounted for relatedness. A SNP, highly correlated $\left(R^{2}>0.99\right)$ with the original SNP, was used as a proxy when the original SNP was not available for outcomes.

\section{Statistics}

We also used MR-Egger to test for potential pleiotropic effects as it may generate correct estimates even when all SNPs are invalid instruments if the assumption of instrument strength independent of direct effect (InSIDE) is satisfied. MR-Egger allows free estimation of the intercept, although further assumptions, such as the independence between instrument strength and direct effects, cannot be easily verified. Average directional pleiotropy across genetic variants was assessed from the $p$-value of the intercept term from MR-Egger [33]. Causal estimates in MR-Egger are less precise than those obtained using inverse variance-weighted (WM) MR [34]. Analysis using MR-Egger has a lower false positive rate but a higher false negative rate than the inverse variance weighted (IVW) method [35]. The WM estimate, as the weighted median of the SNP-specific estimates, provides correct estimates if SNPs accounting for $\geq 50 \%$ of the weight are valid instruments. WM MR allows some variants to be invalid instruments provided at least half are valid instruments. It uses inverse variance weights and bootstrapping to estimate Cls [33].

Further, to assess heterogeneity between individual genetic variants' estimates, we used the $\mathrm{Q}^{\prime}$ heterogeneity statistic [36] and the MR pleiotropy residual sum and outlier (MR-PRESSO) [34] test. The $Q^{\prime}$ statistic uses modified $2^{\text {nd }}$ order weights that are a derivation of a Taylor series expansion and take into account uncertainty in both numerator and denominator of the instrumental variable ratio (this eases the NO-measurement (NOME)-error assumption) [36]. The MR-PRESSO framework relies on the regression of variant-outcome associations on variant-exposure associations and implements a global heterogeneity test by comparing the observed distance (residual sums of squares) of all variants to the regression line with the distance expected under the null hypothesis of no pleiotropy [37]. In case of evidence of horizontal pleiotropy, the test compares individual variants expected and observed distributions to identify outlier variants. We consider as results causal estimates that agreed in direction and magnitude across $M R$ methods, pass nominal significance in inverse variance-weighted $M R$, and did not show evidence of bias from horizontal pleiotropy using heterogeneity tests. MRRAPS (Mendelian randomization using the robust adjusted profile score) is a method for correcting for pleiotropy using robust adjusted profile scores. We used R (version 3.4.2 R Core Team (2017). R: A language and environment for statistical computing. R Foundation for Statistical Computing, Vienna, Austria). 
Mohsen Mazidi, Dimitri P. Mikhailidis, Abbas Dehghan, Jacek Jóźwiak, Adrian Covic, Naveed Sattar, Maciej Banach on behalf of the Lipid and Blood Pressure Meta-analysis Collaboration (LBPMC) Group and the International Lipid Expert Panel (ILEP)

\section{Ethics}

This investigation uses published or publicly available summary data with no involvement of participants in the study. No original data were collected for this manuscript. Ethical approval for each of the studies included in the investigation can be found in the original publications (including informed consent from each subject). The study conforms to the ethical guidelines of the 1975 Declaration of Helsinki.

\section{Systematic review and meta-analysis}

\section{Literature search and study selection}

This meta-analysis was designed, conducted and reported according to Meta-analysis of Observational Studies in Epidemiology (MOOSE) guidelines [38]. The primary exposure of interest was coffee intake, while the primary outcome of interest was risk of CKD. Studies published up to $30^{\text {th }}$ January 2019 (without language restriction) were searched using PubMed, Embase, and Scopus databases; the query syntax of searching is shown in the Supplementary Methods (Supplementary Table SII). This was complemented by hand searches of the reference list of eligible articles, and email correspondences with authors for additional data, where relevant.

After excluding duplicates and based on titles and abstracts, we excluded studies on animals. Eligible studies were selected by using predefined inclusion criteria of case-control, cross-sectional or cohort studies published as original articles to evaluate the risk of CKD among subjects with coffee consumption compared with subjects without coffee consumption. In addition, supplementary hand searching of reference lists of previous reviews or meta-analyses was conducted.

\section{Study selection}

Study selection started with the removal of duplicates, followed by screening of titles and abstracts by two reviewers (MM and JJ). To avoid bias, the reviewers were blinded to the names, qualifications, or the institutional affiliations of the study authors. Agreement between the reviewers was excellent (kappa index: 0.91; $p<0.001$ ). Disagreements were resolved at a meeting between reviewers prior to selected articles being retrieved (a flow chart is available in Supplementary Figure S1).

We included studies if they met all the following criteria: (1) the studies of interest were on coffee intake; (2) the studies were population-based cohort studies and reported CKD data; (3) relative risk (RR), hazard ratio (HR) or OR estimates with 95\% Cls adjusted for multivariable factors were available or were able to be calculated; (4) original articles with full texts in English.

Studies were excluded according to the following criteria: (1) reviews, letters, unpublished data, or comments; (2) those published in languages other than English; (3) not population-based cohort studies; (4) RR, HR or OR estimates with $95 \% \mathrm{Cls}$ were not available or were not be able to be calculated. Narrative reviews, comments, opinion pieces, methodological papers, editorials, letters or any other publications lacking primary data and/or explicit method descriptions were also excluded.

\section{Data extraction and management}

The full texts of studies meeting the inclusion criteria were retrieved and screened to determine eligibility by 2 reviewers (MM, JJ). The study quality assessment was performed according to the Newcastle-Ottawa Scale (NOS, Supplementary Table SIII) [39]. The following perspectives were considered: representativeness of the exposed cohort; dietary survey methods of flavonoid intake; comparability of cohorts (adjustment for important confounders); assessment of outcome; duration of follow-up; and adequacy of follow-up. By evaluation of selection, comparability and outcome, the rating system scores for studies range from 0 (highest degree of bias) to 9 (lowest degree of bias). Following assessment of methodological quality, 2 reviewers (MM \& JJ) extracted data using a purpose-designed data extraction form and independently summarized what they consider to be the most important results from each study. These summaries were compared, and any differences of opinion were resolved by discussion and consultation with a third reviewer (MB). Any further calculations on study data considered necessary were conducted by the first reviewer and checked by the second reviewer. Information extracted from each eligible study included the following items: author, year and reference, country, region/cohort, coffee consumption measurement, design, sample size, definition of CKD, main confounders (Supplementary Table SIV).

\section{Data synthesis and statistical analyses}

For studies that reported results from different multivariable-adjusted models, the model including the most confounding factors was extracted for the meta-analysis. The random-effect model was applied to calculate pooled RRs, $95 \% \mathrm{Cl}$ and $p$-value for heterogeneity. Effect estimates comparing the highest with the lowest intake category were combined across studies to generate the summary associations. The extent of heterogeneity across studies was examined using the 
$R^{2}$ test [40-42] and $I^{2}>50 \%$ together with $p<0.05$ two-sided indicated significant heterogeneity [40-42]. To examine whether the present results were affected by a single study, we conducted a sensitivity analysis by excluding one study at a time.

\section{Publication bias}

Potential publication bias was explored using visual inspection of Begg's funnel plot asymmetry, Begg's rank correlation and Egger's weighted regression tests. The Duval and Tweedie trim method was used to adjust the analysis for the effects of publication bias [43].

\section{Results}

\section{NHANES sata}

Of the 18,436 participants included in the analyses, $6.9 \%$ had prevalent CKD (based on eGFR). The characteristics of participants overall and across caffeine quartiles are summarized in Table I. The caffeine intake for the general population was $131.1 \pm 1.1 \mathrm{mg}$ and was higher for men compared to women $(149.1 \pm 2.2$ vs. 114.2 \pm 1.7 respectively) $(p<0.001)$. Mean age across the caffeine intake was in the range 44.2-50.1 years, while the proportion of men decreased from $43.8 \%$ to $57.5 \%$ across increasing quartiles of caffeine. Across increasing caffeine quartiles, the proportion of non-Hispanic White (the largest ethnic group) increased from $32.8 \%$ to $70.0 \%$; the proportions of Non-Hispanic Black decreased from $20.7 \%$ to $11.7 \%$; and Mexican-American followed the same trend (reduction from $33.3 \%$ to $9.1 \%$, $p<0.001$, Supplementary Table SI). The proportion of participants with prevalent low eGFR, albuminuria or CKD systematically decreased across increasing quartiles of caffeine (all $p<0.001$ for linear trend, Table I). Proportions of participants with CKD, by caffeine quartile, were $9.4 \%$ in the first (lowest) quartile, $7.8 \%$ in the second, $5.8 \%$ in the third and $5.2 \%$ in the top quartile (Table I).

Adjusted mean levels of renal function markers by quartile of caffeine are shown in Table II. For increasing quartiles for caffeine intake, mean urine albumin did not change significantly (2.20 to 2.17 $\mathrm{mg} / \mathrm{l}, p=0.239)$; the same was observed for eGFR (91.2 to $89.6 \mathrm{ml} / \mathrm{min} / 1.73 \mathrm{~m}^{2}, p=0.415$, Table II). Log ACR also remained stable with no significant change across the quartiles of caffeine (2.14 to $2.16 \mathrm{mg} / \mathrm{g}, p=0.352)$. In age, sex and race adjusted logistic regressions, compared with the lowest quartile of caffeine, the odds ratio $(95 \% \mathrm{Cl})$ was

Table I. Descriptive characteristics of participants across quartiles of caffeine

\begin{tabular}{|c|c|c|c|c|c|}
\hline \multirow[t]{2}{*}{ Characteristics } & \multicolumn{4}{|c|}{ Quartiles of caffeine } & \multirow[t]{3}{*}{$P$-value } \\
\hline & First & Second & Third & Fourth & \\
\hline Median $\left(25^{\text {th }}-75^{\text {th }}\right)[\mathrm{mg}]$ & $11(7-19)$ & $51(34-72)$ & $142(112-171)$ & $329(260-462)$ & \\
\hline Number of participants & 4609 & 4611 & 4608 & 4608 & \\
\hline Gender (\%): & & & & & $<0.001$ \\
\hline Male & 43.8 & 44.7 & 48.9 & 57.5 & \\
\hline Female & 56.2 & 55.3 & 51.1 & 42.5 & \\
\hline Age [years] & $44.2 \pm 0.3$ & $45.6 \pm 0.3$ & $47.4 \pm 0.2$ & $50.1 \pm 0.2$ & $<0.001$ \\
\hline Race (\%): & & & & & $<0.001$ \\
\hline White (non-Hispanic) & 32.8 & 36.7 & 49.3 & 70.0 & \\
\hline Non-Hispanic Black & 20.7 & 24.5 & 19.5 & 11.7 & \\
\hline Mexican-American & 33.3 & 23.9 & 17.2 & 9.1 & \\
\hline Income to poverty $(n)$ & $2.3 \pm 0.02$ & $2.3 \pm 0.02$ & $2.5 \pm 0.02$ & $2.8 \pm 0.02$ & $<0.001$ \\
\hline $\mathrm{BMI}\left[\mathrm{kg} / \mathrm{m}^{2}\right]$ & $28.8 \pm 0.1$ & $28.6 \pm 0.1$ & $28.9 \pm 0.1$ & $28.8 \pm 0.1$ & 0.253 \\
\hline Energy intake [kcal/day] & $1970.2 \pm 14.2$ & $2053.2 \pm 15.3$ & $2086.7 \pm 14.2$ & $2339.2 \pm 15.9$ & $<0.001$ \\
\hline Alcohol intake [g/day] & $9.5 \pm 0.4$ & $8.9 \pm 0.3$ & $9.6 \pm 0.5$ & $12.1 \pm 0.4$ & $<0.001$ \\
\hline Triglyceride [mg/dl] & $144.3 \pm 1.9$ & $145.5 \pm 2.0$ & $157.4 \pm 1.8$ & $160.2 \pm 2.3$ & $<0.001$ \\
\hline Type 2 diabetes (\%) & 17.9 & 18.4 & 19.1 & 17.0 & $<0.001$ \\
\hline Hypertension (\%) & 10.9 & 10.1 & 10.5 & 11.3 & $<0.001$ \\
\hline Low eGFR (\%) & 9.4 & 7.8 & 5.8 & 5.2 & $<0.001$ \\
\hline Albuminuria (\%) & 9.8 & 10.1 & 11.5 & 9.7 & $<0.001$ \\
\hline $\begin{array}{l}\text { CKD (\%) [low eGFR or } \\
\text { albuminuria] }\end{array}$ & 16.6 & 13.9 & 12.2 & 11.0 & $<0.001$ \\
\hline
\end{tabular}

Value expressed as mean and S.E.M. or percentage. CKD - chronic kidney disease, eGFR - estimated glomerular filtration rate. 
Mohsen Mazidi, Dimitri P. Mikhailidis, Abbas Dehghan, Jacek Jóźwiak, Adrian Covic, Naveed Sattar, Maciej Banach on behalf of the Lipid and Blood Pressure Meta-analysis Collaboration (LBPMC) Group and the International Lipid Expert Panel (ILEP)

Table II. Adjusted (age, sex, race, alcohol intake, energy intake, smoking, BMI, HTN, TG and DM) mean levels of markers of CKD across quartiles of caffeine

\begin{tabular}{|lccccc|}
\hline Characteristics & \multicolumn{4}{c}{ Quartiles of caffeine } & \multirow{2}{*}{ P-value } \\
\cline { 2 - 5 } & First & Second & Third & Fourth & \\
\hline Number of participants $(n)$ & 4609 & 4611 & 4608 & 4608 \\
\hline Log urine albumin $[\mathrm{mg} / \mathrm{l}]$ & $2.20 \pm 0.02$ & $2.16 \pm 0.02$ & $2.19 \pm 0.02$ & $2.17 \pm 0.02$ & 0.239 \\
\hline Serum creatinine $[\mathrm{mg} / \mathrm{dl}]$ & $0.89 \pm 0.003$ & $0.90 \pm 0.004$ & $0.91 \pm 0.002$ & $0.88 \pm 0.003$ & 0.234 \\
\hline Log ACR $[\mathrm{mg} / \mathrm{g}]$ & $2.14 \pm 0.02$ & $2.10 \pm 0.02$ & $2.11 \pm 0.02$ & $2.16 \pm 0.02$ & 0.352 \\
\hline eGFR $\left[\mathrm{ml} / \mathrm{min} / 1.73 \mathrm{~m}^{2}\right]$ & $91.2 \pm 0.7$ & $92.8 \pm 0.4$ & $90.2 \pm 0.5$ & $89.6 \pm 0.3$ & 0.415 \\
\hline
\end{tabular}

Values (from analysis of covariance) expressed as mean \pm S.E.M. eGFR - estimated glomerular filtration rate, ACR - urine albumin/ creatinine ratio.

$0.76(0.55-1.09)$ for Q2, $1.03(0.95-1.08)$ for $\mathrm{Q} 3$ and $0.97(0.86-1.12)$ for $\mathrm{Q} 4(p=0.355$ for trend, CKD diagnosed by eGFR). In logistic regression models adjusted for age, sex, race, income to poverty, alcohol intake, energy intake, smoking, BMI, HTN, TG and T2DM, compared with the lowest quartile of caffeine, the odds ratio $(95 \% \mathrm{Cl})$ of low eGFR was 1.00 (0.96-1.04) for Q2, 0.99 (0.96-1.03) for Q3 and $1.02(0.94-1.10)$ for Q4 ( $p=0.745$ for trend).

\section{Mendelian randomization}

The list of all instruments associations for coffee intake is shown in Supplementary Table SI. The results, expressed as the $\beta$-coefficient for outcomes of interest per one cup per day increase in coffee consumption, demonstrate no effect on CKD (IVW: $\beta=-0.0191$, SE $=0.069, p=0.781$ ) or eGFR (overall $=\mathrm{IVW}: \beta=-0.0005, \mathrm{SE}=0.005$, $p=0.926$; both for DM: IVW: $\beta=-0.006, \mathrm{SE}=0.009$, $p=0.478$, and non-DM subjects: IVW: $\beta=-6.772$, $\mathrm{SE}=0.006, p=0.991$, Table III). Results of the forest and scatter plots for each outcome are shown in Figures 1 to 4; further analysis of MR-PRESSO did not identify any outlier. As can be seen in Table III, pleiotropy bias did not indicate any chance of pleiotropy for all 4 levels of our outcomes; furthermore, the results of the MR-RAPS were identical to the

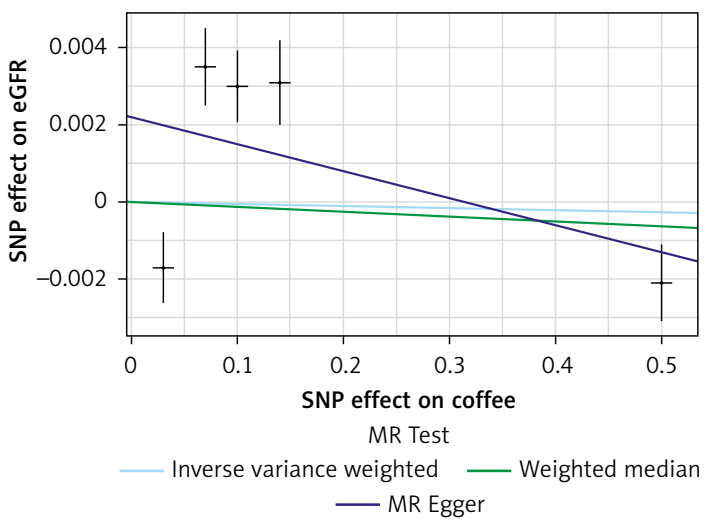

Figure 1. Scatter plots of genetic associations with coffee/caffeine level against genetic associations with eGFR. The slopes of each line represent causal associations for each method
IVW prediction, which again indicated no chance of pleiotropy (Table III). The heterogeneity tests were significant for all levels of outcomes (Figures 1 and 2) but not for the GFR between DM populations (MR-Egger: $\mathrm{Q}=5.579, p=0.133$, Table III). All but three of the coffee-increasing alleles of the five SNPs with a genome-wide significant association with coffee consumption were positively associated with eGFR (rs17685, rs4410790, rs2472297), and the association with rs7800944, near the gene encoding MLXIPL (promoters of triglyceride synthesis genes), was statistically significant and negatively affected eGFR (Figure 3). With regard to CKD, just a single SNP had a significant effect, and it was inversely related (rs17685, making the enzyme cytochrome P450 oxidoreductase, Figure 4).

\section{Meta-analysis and systematic review}

Overviews of key characteristics of the 6 studies are shown in Supplementary Table SIV. A total of 42,051 participants were included in the analysis. All the studies involved both men and women. They were performed in various countries, including: Italy [9], Japan [11, 12], Korea [10, 13] and the USA. In five of them CKD was diagnosed as GFR $<60 \mathrm{ml} / \mathrm{min} / 1.73 \mathrm{~m}^{2}$ [10-12] and in one of them it was renal resistive index $(\mathrm{RRI})>0.65$ (renal color

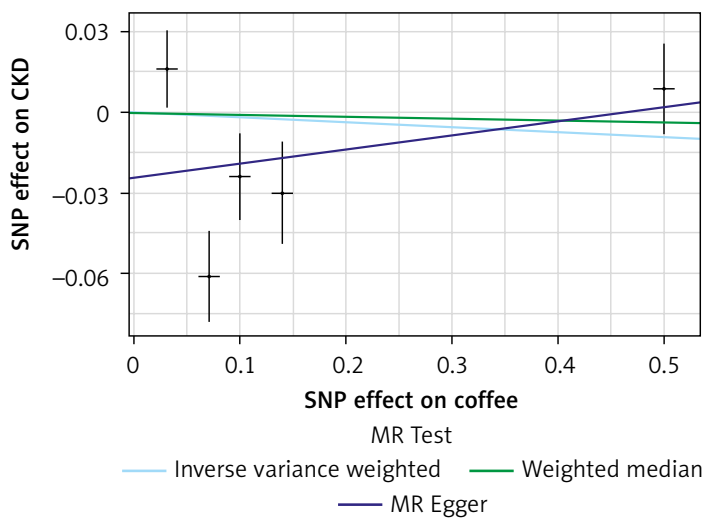

Figure 2. Scatter plots of genetic associations with coffee/caffeine level against genetic associations with CKD. The slopes of each line represent causal associations for each method 
Doppler echocardiography was used to evaluate RRI) [9]. Results of quality assessment are shown in Supplementary Table SIII, with all studies scoring $\geq 7$.

\section{Coffee consumption and risk of CKD}

Based on the meta-analysis of available studies we showed that coffee consumption was not significantly associated with CKD (OR $=0.85$, $95 \% \mathrm{Cl}: 0.71-1.02, p=0.090, n=6$ studies, $R^{2}=0.32$, Supplementary Figure S2).

\section{Sensitivity analysis}

In leave-one-out sensitivity analyses, the pooled effect estimates remained similar for the effect on coffee consumption for CKD (OR $=0.85$, $95 \% \mathrm{Cl}: 0.71-1.02)$. This stability confirms that the significant difference between the studied groups is the overall effect of all included studies.

\section{Publication bias}

Visual inspection of the funnel plot symmetry suggested no potential publication bias for the comparison of coffee consumption and CKD. Moreover, Egger's linear regression indicated no publication bias (intercept $=-1.4$, two-tailed $p=$ 0.421), as well as the Begg's rank correlation test (Kendall's $\tau$ with continuity correction $=1.00$, two tailed $p=0.362$ ). After adjustment of the effect size for potential publication bias using the 'trim and fill' correction, no potentially missing studies were imputed in the funnel plot $(O R=0.86$, 95\% Cl: 0.70-1.07).

\section{Discussion}

The present study, by applying three different methods, examined the association between caffeine intake and prevalent CKD. We used the largest, nationally representative population of adult Americans to evaluate the link between caffeine and renal function. Further, we applied the largest available GWAS to obtain unconfounded estimates of the association between genetically instrumented coffee intake, eGFR and CKD. Further, we systematically searched and pooled the studies. Individual-level data and meta-analysis indicated that caffeine/coffee intake is not associated with likelihood of CKD and kidney function tests. MR data confirmed the main findings and revealed no causal effect of coffee intake on eGFR and CKD.

Available information on the potential association between renal function and coffee is controversial [8-12]. In a cross-sectional study in Italy $(n=221)$, in which coffee consumption was assessed by an interviewer-administered food fre-

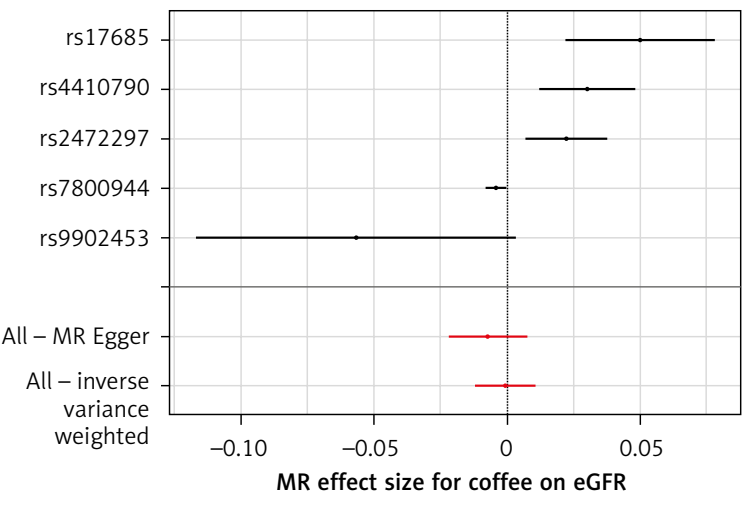

Figure 3. Forest plots of genetic associations with coffee/caffeine level against genetic associations with eGFR. The slopes of each line represent causal associations for each method

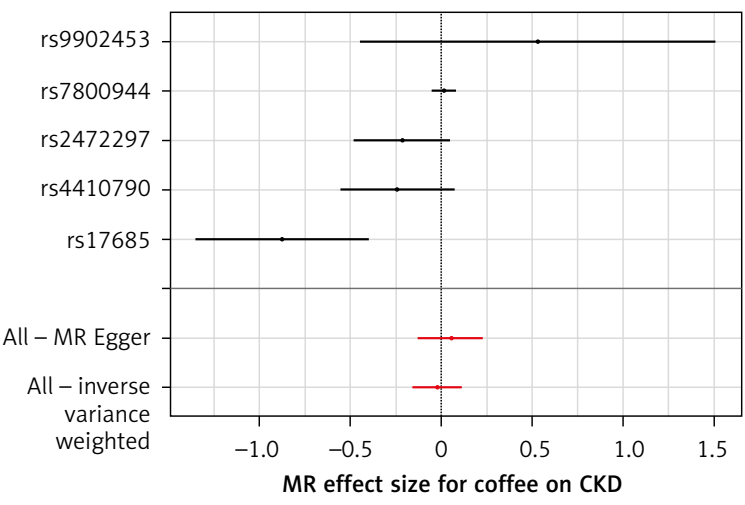

Figure 4. Forest plots of genetic associations with coffee/caffeine level against genetic associations with CKD. The slopes of each line represent causal associations for each method

quency questionnaire, the authors reported a significant inverse association between coffee intake and risk of CKD (OR $=0.46,95 \% \mathrm{Cl}: 0.24-0.89)$ [9]. Kim et al. in their study performed in Korea $(n=2,673)$, on women aged $35-64$ years from the $4^{\text {th }}$ Korean National Health and Nutrition Examination Surveys, also reported the positive effect of caffeine intake on CKD prevalence $(O R=0.59$, 95\% Cl: 0.37-0.95) [10]. Two other studies have reported no association between caffeine/coffee and CKD risk [11, 12] - Pham et al., in a cross-sectional study with 11,662 participants, reported no significant association between coffee intake and likelihood of CKD (OR = 1.01, 95\% Cl: 0.84-1.21) [12]; similar results were observed in another Japanese cross-sectional study $(n=342 ; \mathrm{OR}=0.74$, 95\% Cl: 0.30-1.85) [11]. In a prospective study that followed 4,722 participants aged 26-65 year for 15 years, Herber-Gast et al. found that coffee consumption was associated with a slightly higher eGFR, particularly in subjects aged $\geq 46$ years. The authors concluded that the absence of an association with eGFR changes suggests that the higher eGFR among coffee consumers is unlikely to be a result of glomerular hyperfiltration [44]. A me- 
Mohsen Mazidi, Dimitri P. Mikhailidis, Abbas Dehghan, Jacek Jóźwiak, Adrian Covic, Naveed Sattar, Maciej Banach on behalf of the Lipid and Blood Pressure Meta-analysis Collaboration (LBPMC) Group and the International Lipid Expert Panel (ILEP)

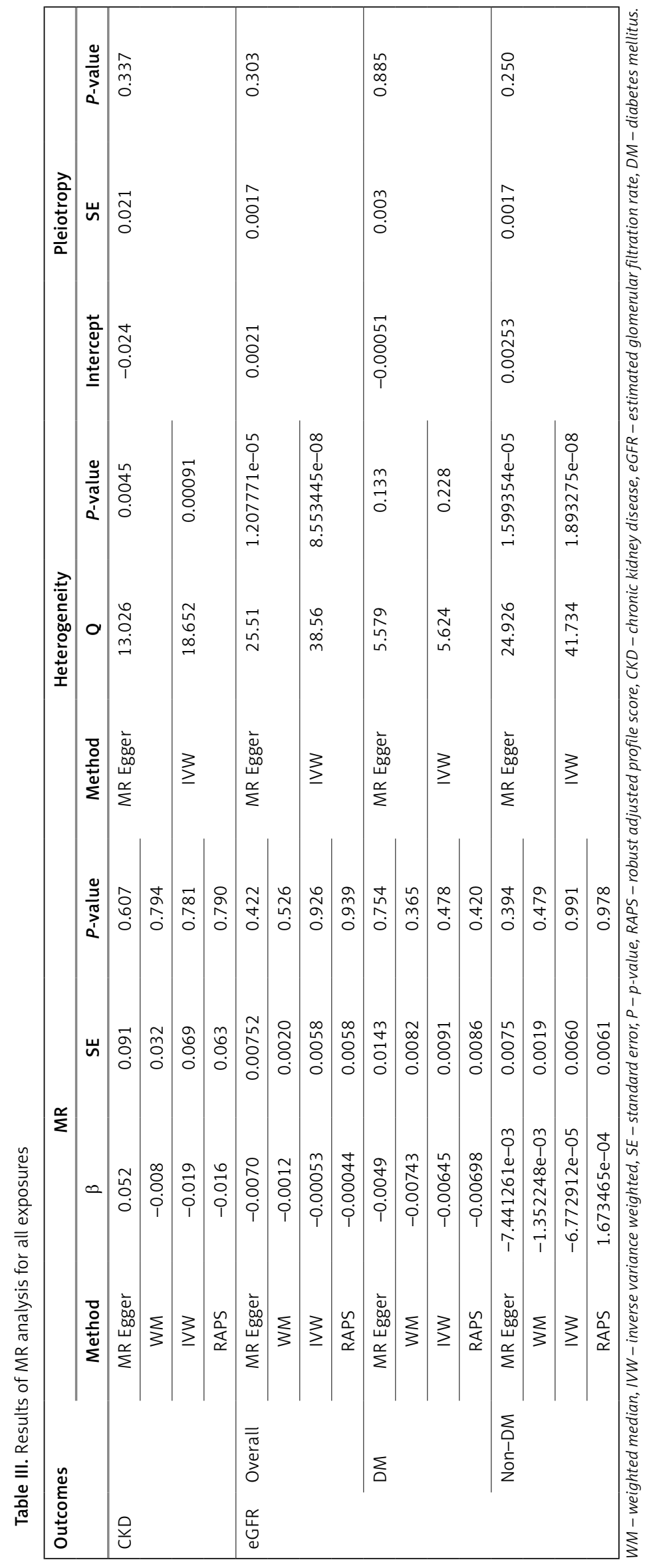


ta-analysis of 4 studies reported no significant association between coffee consumption and the risk of CKD (OR $=0.71,95 \% \mathrm{Cl}: 0.47-1.08)$ [8]. Taking into account mostly positive data of moderate coffee consumption with the risk of cardiovascular disease, but also the link between coffee/caffeine intake and blood pressure increase, and especially very recent data suggesting a significant inverse association between caffeine intake and all-cause mortality in CKD patients [45], one might have expected a potential significant impact on CKD.

There are indeed very limited data on the association between caffeine intake and renal function parameters, which makes it difficult to compare our findings. In a cross-sectional study between 11,662 Japanese men and women aged 49-76 the authors reported that caffeine intake was weakly inversely associated with serum uric acid (SUA) in the age-adjusted model [12], while in another study on 14,758 participants aged $>20$ years, the authors reported no link between caffeine intake and SUA [46]. Hence the results concerning the potential impact of caffeine intake on kidney functions tests seems to be still controversial. However, taking into account our data, it seems that, similarly to the link between caffeine intake and CKD prevalence, there is also no significant link with the parameters characterizing kidney function [47-57].

The present analysis has some strengths to be emphasized. This is the largest data analysis of the association of kidney disease with caffeine intake. Participants were a random sample of individuals drawn from the general population, and therefore the results can be extrapolated to the general US population. Because data collection was performed on all days of the week in NHANES, the potential for day-specific information bias is very low $[47,48]$. We evaluated our findings by applying MR, which is known to be a powerful tool for detection of a causal impact.

Our findings, however, also have to be considered in the context of some study limitations. First, the cross-sectional nature of the data does not allow for direct inference about causality. Second, it is well known that a single 24-hour diet recall interview is not ideal for characterizing an individual's long-term habitual intake [49, 50]. Third, individuals with renal impairment may be less likely to drink caffeinated drinks, which may have led to underestimation in our results. As with any meta-analysis, internal validity relies on the quality of individual studies. Several limitations can be identified in this regard.

In conclusion, the present study, by applying three different methods to investigate the association between caffeine intake and prevalent CKD, confirmed no impact of caffeine consumption on
CKD risk and kidney function tests. MR data additionally revealed no causal effect of coffee intake on eGFR and CKD.

\section{Acknowledgments}

The material presented in this manuscript is original and has not been submitted for publication elsewhere. The results of this analysis were presented during the European Society of Cardiology (ESC) Congress 2019 in Paris, France.

\section{Conflict of interest}

DPM has given talks and attended conferences sponsored by MSD, AstraZeneca and Libytec; MB speakers bureau: Amgen, Esperion, Herbapol, Kogen, KRKA, Novartis, Polpharma, Sanofi-Aventis, Servier, Teva, Viatris and Zentiva; consultant to Akcea, Amgen, Daichii Sankyo, Esperion, Freia Pharmaceuticals, Polfarmex, Sanofi-Aventis; Grants from Amgen, Viatris, Sanofi and Valeant; all other authors have no conflict of interest to declare.

\section{References}

1. Levey AS, Coresh J. Chronic kidney disease. Lancet 2012; 379: 165-80.

2. Miyamoto T, Carrero JJ, Stenvinkel P. Inflammation as a risk factor and target for therapy in chronic kidney disease. Curr Opin Nephrol Hypertens 2011; 20: 662-8.

3. Leung KC, Tonelli M, James MT. Chronic kidney disease following acute kidney injury-risk and outcomes. Nature Rev Nephrol 2013; 9: 77-85.

4. Giugliano D, Ceriello A, Esposito K. The effects of diet on inflammation: emphasis on the metabolic syndrome. J Am Coll Cardiol 2006; 48: 677-85.

5. Mazidi M, Gao HK, Kengne AP. Food Patterns are associated with likelihood of CKD in US adults. Sci Rep 2018; 8: 10696.

6. Mazidi M, Shivappa N, Wirth MD, Hebert JR, Kengne AP. Greater Dietary Inflammatory Index score is associated with higher likelihood 2 of chronic kidney disease. $\mathrm{Br}$ J Nutr 2018; 120: 204-9.

7. Liu F, Wang X, Wu G, et al. Coffee consumption decreases risks for hepatic fibrosis and cirrhosis: a meta-analysis. PLoS One 2015; 10: e0142457.

8. Wijarnpreecha K, Thongprayoon C, Thamcharoen N, Panjawatanan P, Cheungpasitporn W. Association of coffee consumption and chronic kidney disease: a meta-analysis. Int J Clin Pract 2017; 71: doi: 10.1111/ ijcp.12919.

9. Trovato GM, Pirri C, Martines GF, Trovato F, Catalano D. Coffee, nutritional status, and renal artery resistive index. Renal Fail 2010; 32: 1137-47.

10. Kim BH, Park YS, Noh HM, Sung JS, Lee JK. Association between coffee consumption and renal impairment in Korean women with and without diabetes: analysis of the Fourth Korea National Health and Nutrition Examination Survey in 2008. Korean J Fam Med 2013; 34: 265-71.

11. Nakajima K, Hirose K, Ebata M, Morita K, Munakata H. Association between habitual coffee consumption and normal or increased estimated glomerular filtration rate in apparently healthy adults. Br J Nutr 2010; 103: 149-52. 
12. Pham NM, Yoshida D, Morita M, et al. The relation of coffee consumption to serum uric acid in Japanese men and women aged 49-76 years. J Nutr Metabol 2010; 2010: 930757

13. Jhee $\mathrm{JH}, \mathrm{Nam} \mathrm{KH}$, An SY, et al. Effects of coffee intake on incident chronic kidney disease: a community-based prospective cohort study. Am J Med 2018; 131: 148290.e3.

14. Smith GD, Ebrahim S. 'Mendelian randomization': can genetic epidemiology contribute to understanding environmental determinants of disease? Int J Epidemiol 2003; 32: 1-22.

15. National Center for Health Statistics. http://www.cdc gov/nchs/nhanes.htm.

16. Needham BL, Adler N, Gregorich S, et al. Socioeconomic status, health behavior, and leukocyte telomere length in the National Health and Nutrition Examination Survey, 1999-2002. Soc Sci Med (1982) 2013; 85: 1-8.

17. Tighe P, Duthie G, Vaughan N, et al. Effect of increased consumption of whole-grain foods on blood pressure and other cardiovascular risk markers in healthy mid dle-aged persons: a randomized controlled trial. Am J Clin Nutr 2010; 92: 733-40.

18. Mazidi M, Michos ED, Banach M. The association of telomere length and serum 25-hydroxyvitamin D levels in US adults: the National Health and Nutrition Examination Survey. Arch Med Sci 2017; 13: 61-5.

19. http://www.cdc.gov/NCHS/data/nhanes/ nhanes 09 10/CRP F met.pdf. [accessed 19.08.18].

20. Selvin E, Manzi J, Stevens LA, et al. Calibration of serum creatinine in the National Health and Nutrition Examination Surveys (NHANES) 1988-1994, 1999-2004. Am J Kidney Dis 2007; 50: 918-26.

21. Chavers BM, Simonson J, Michael AF. A solid phase fluorescent immunoassay for the measurement of human urinary albumin. Kidney Int 1984; 25: 576-8.

22. Levey AS, Stevens LA, Schmid $\mathrm{CH}$, et al. A new equation to estimate glomerular filtration rate. Ann Intern Med 2009; 150: 604-12.

23. Kidney Disease: Improving Global Outcomes (KDIGO). Chapter 1: Definition and classification of CKD. Kidney Int Suppl (2011) 2013; 3: 19-62.

24. Nwankwo T, Yoon SS, Burt V, Gu Q. Hypertension among adults in the United States: National Health and Nutrition Examination Survey, 2011-2012. NCHS Data Brief 2013; (133): 1-8

25. Ahluwalia N, Andreeva VA, Kesse-Guyot E, Hercberg S. Dietary patterns, inflammation and the metabolic syndrome. Diabetes Metab 2013; 39: 99-110.

26. Ahluwalia N, Dwyer J, Terry A, Moshfegh A, Johnson C. Update on NHANES Dietary Data: focus on collection, release, analytical considerations, and uses to inform public policy. Adv Nutr 2016; 7: 121-34.

27. Moshfegh AJ, Rhodes DG, Baer DJ, et al. The US Department of Agriculture Automated Multiple-Pass Method reduces bias in the collection of energy intakes. Am J Clin Nutr 2008; 88: 324-32.

28. Drewnowski A, Rehm CD. Sources of caffeine in diets of US children and adults: trends by beverage type and purchase location. Nutrients 2016; 8: 154.

29. NHANES Analytic And Reporting Guidelines. https:// www.cdc.gov/nchs/data/nhanes/nhanes_03_04/ nhanes analytic guidelines dec 2005.pdf.

30. Cornelis MC, Byrne EM, Esko T, et al. Genome-wide meta-analysis identifies six novel loci associated with habitual coffee consumption. Mol Psychiatry 2015; 20: 647-56.
31. Lawlor DA, Harbord RM, Sterne JA, Timpson N, Davey Smith $\mathrm{G}$. Mendelian randomization: using genes as instruments for making causal inferences in epidemiology. Statistics Med 2008; 27: 1133-63.

32. Pattaro C, Teumer A, Gorski M, et al. Genetic associations at 53 loci highlight cell types and biological pathways relevant for kidney function. Nat Commun 2016; 7: 10023.

33. Bowden J, Davey Smith G, Haycock PC, Burgess S. Consistent estimation in mendelian randomization with some invalid instruments using a weighted median estimator. Genet Epidemiol 2016; 40: 304-14.

34. Bowden J, Davey Smith G, Burgess S. Mendelian randomization with invalid instruments: effect estimation and bias detection through Egger regression. Int J Epidemiol 2015; 44: 512-25.

35. Burgess S, Bowden J, Fall T, Ingelsson E, Thompson SG. Sensitivity analyses for robust causal inference from mendelian randomization analyses with multiple genetic variants. Epidemiology 2017; 28: 30-42.

36. Bowden J, Del Greco MF, Minelli C, Davey Smith G, Sheehan $\mathrm{N}$, Thompson J. A framework for the investigation of pleiotropy in two-sample summary data Mendelian randomization. Stat Med 2017; 36: 1783-802.

37. Verbanck M, Chen CY, Neale B, Do R. Detection of widespread horizontal pleiotropy in causal relationships inferred from Mendelian randomization between complex traits and diseases. Nature Genet 2018; 50: 693-8.

38. Stroup DF, Berlin JA, Morton SC, et al. Meta-analysis of observational studies in epidemiology: a proposal for reporting. Meta-analysis Of Observational Studies in Ep idemiology (MOOSE) group. JAMA 2000; 283: 2008-12.

39. Stang A. Critical evaluation of the Newcastle-Ottawa scale for the assessment of the quality of nonrandomized studies in meta-analyses. Eur J Epidemiol 2010; 25 603-5.

40. Ferretti G, Bacchetti T, Sahebkar A. Effect of statin therapy on paraoxonase-1 status: a systematic review and meta-analysis of 25 clinical trials. Progress Lipid Res 2015; 60: 50-73.

41. Mazidi M, Mikhailidis DP, Sattar N, et al.; International Lipid Expert Panel (ILEP) \& Lipid and Blood Pressure Meta-analysis Collaboration (LBPMC) Group. Association of types of dietary fats and all-cause and cause-specific mortality: a prospective cohort study and meta-analysis of prospective studies with 1,164,029 participants. Clin Nutr 2020; 39: 3677-86

42. Sahebkar A, Serban MC, Mikhailidis DP, et al. Head-tohead comparison of statins versus fibrates in reducing plasma fibrinogen concentrations: a systematic review and meta-analysis. Pharmacol Res 2016; 103: 236-52.

43. Duval S, Tweedie R. Trim and fill: a simple funnel-plotbased method of testing and adjusting for publication bias in meta-analysis. Biometrics 2000; 56: 455-63.

44. Herber-Gast GC, van Essen $\mathrm{H}$, Verschuren WM, et al. Coffee and tea consumption in relation to estimated glomerular filtration rate: results from the population-based longitudinal Doetinchem Cohort Study. Am J Clin Nutrition 2016; 103: 1370-7.

45. Bigotte Vieira M, Magrico R, Viegas Dias C, Leitao L, Neves JS. Caffeine consumption and mortality in chronic kidney disease: a nationally representative analysis. Nephrol Dialysis Transplant 2019; 34: 974-80.

46. Choi HK, Curhan G. Coffee, tea, and caffeine consumption and serum uric acid level: the third national health and nutrition examination survey. Arthritis Rheum 2007; 57: 816-21. 
47. Tooze JA, Midthune D, Dodd KW, et al. A new statistical method for estimating the usual intake of episodically consumed foods with application to their distribution. J Am Diet Assoc 2006; 106: 1575-87.

48. Guenther PM, Ding EL, Rimm EB. Alcoholic beverage consumption by adults compared to dietary guidelines: results of the National Health and Nutrition Examination Survey, 2009-2010. J Acad Nutr Diet 2013; 113: 546-50.

49. Hebert JR, Hurley TG, Steck SE, et al. Considering the value of dietary assessment data in informing nutrition-related health policy. Adv Nutr 2014; 5: 447-55.

50. Ma Y, Olendzki BC, Pagoto SL, et al. Number of 24-hour diet recalls needed to estimate energy intake. Ann Epidemiol 2009; 19: 553-9.

51. Penson P, Serban MC, Ursoniu S, Banach M; Lipid and Blood Pressure Meta-analysis Collaboration (LBPMC) Group. Does coffee consumption alter plasma lipoprotein(a) concentrations? A systematic review. Crit Rev Food Sci Nutr 2018; 58: 1706-14.

52. Giglio RV, Patti AM, Cicero AFG, et al. Polyphenols: potential use in the prevention and treatment of cardiovascular diseases. Curr Pharm Des 2018; 24: 239-58.

53. Kaczmarczyk-Sedlak I, Folwarczna J, Sedlak L, et al. Effect of caffeine on biomarkers of oxidative stress in lenses of rats with streptozotocin-induced diabetes. Arch Med Sci 2019; 15: 1073-80.

54. Rysz J, Franczyk B, Banach M, Gluba-Brzozka A. Hypertension - current natural strategies to lower blood pressure. Curr Pharm Des 2017; 23: 2453-61.

55. Mazidi M, Shekoohi N, Katsiki N, Banach M. Omega-6 fatty acids and the risk of cardiovascular disease: insights from a systematic review and meta-analysis of randomized controlled trials and a Mendelian randomization study. Arch Med Sci 2021; 18: 466-79.

56. Banach M, Burchardt P, Chlebus K, et al. PoLA/CFPiP/ $\mathrm{PCS} / \mathrm{PSLD} / \mathrm{PSD} / \mathrm{PSH}$ guidelines on diagnosis and therapy of lipid disorders in Poland 2021. Arch Med Sci 2021; 17: 1447-547.

57. Mazidi M, Shekoohi N, Katsiki N, Rakowski M, Mikhailidis DP, Banach M. Serum anti-inflammatory and inflammatory markers have no causal impact on telomere length: a Mendelian randomization study. Arch Med Sci 2021; 17: 739-51. 